\title{
AKT1 and MYC Induce Distinctive Metabolic Fingerprints in Human Prostate Cancer
}

\author{
Carmen Priolo ${ }^{1}$, Saumyadipta Pyne ${ }^{1}$, Joshua Rose ${ }^{1}$, Erzsébet Ravasz Regan², Giorgia Zadra ${ }^{1}$, \\ Cornelia Photopoulos ${ }^{1}$, Stefano Cacciatore ${ }^{1}$, Denise Schultz ${ }^{3}$, Natalia Scaglia ${ }^{1}$, Jonathan McDunn ${ }^{4}$, \\ Angelo M. De Marzo ${ }^{3}$, and Massimo Loda ${ }^{1,5,6,7}$
}

\begin{abstract}
Cancer cells may overcome growth factor dependence by deregulating oncogenic and/or tumor-suppressor pathways that affect their metabolism, or by activating metabolic pathways de novo with targeted mutations in critical metabolic enzymes. It is unknown whether human prostate tumors develop a similar metabolic response to different oncogenic drivers or a particular oncogenic event results in its own metabolic reprogramming. Akt and Myc are arguably the most prevalent driving oncogenes in prostate cancer. Mass spectrometry-based metabolite profiling was performed on immortalized human prostate epithelial cells transformed by AKT1 or MYC, transgenic mice driven by the same oncogenes under the control of a prostatespecific promoter, and human prostate specimens characterized for the expression and activation of these oncoproteins. Integrative analysis of these metabolomic datasets revealed that AKT1 activation was associated with accumulation of aerobic glycolysis metabolites, whereas MYC overexpression was associated with dysregulated lipid metabolism. Selected metabolites that differentially accumulated in the MYC-high versus AKT1-high tumors, or in normal versus tumor prostate tissue by untargeted metabolomics, were validated using absolute quantitation assays. Importantly, the AKT1/MYC status was independent of Gleason grade and pathologic staging. Our findings show how prostate tumors undergo a metabolic reprogramming that reflects their molecular phenotypes, with implications for the development of metabolic diagnostics and targeted therapeutics. Cancer Res; 74(24); 7198-204. (C2014 AACR.
\end{abstract}

\section{Introduction}

Metabolomics allows unbiased identification of subtle changes in metabolite profiles as affected by signaling pathways or genetic factors (1-3). Metabolic alterations may represent the integration of genetic regulation, enzyme activity, and metabolic reactions. In addition, because the known

${ }^{1}$ Department of Medical Oncology, Dana-Farber Cancer Institute, Brigham and Women's Hospital, Boston, Massachusetts. ${ }^{2}$ Department of Medicine, Beth Israel Deaconess Medical Center, Harvard Medical School, Boston, Massachusetts. ${ }^{3}$ Department of Pathology, Johns Hopkins University, Baltimore, Maryland. ${ }^{4}$ Metabolon Inc., Durham, North Carolina. ${ }^{5}$ Department of Pathology, Brigham and Women's Hospital, Boston, Massachusetts. ${ }^{6}$ The Broad Institute, Cambridge, Massachusetts. ${ }^{7}$ Division of Cancer Studies, King's College London, United Kingdom.

Note: Supplementary data for this article are available at Cancer Research Online (http://cancerres.aacrjournals.org/)

Current address for C. Priolo: Division of Pulmonary and Critical Care Medicine, Department of Medicine, Brigham and Women's Hospital, Harvard Medical School, Boston, Massachusetts; current address for S. Pyne: C.R. Rao Advanced Institute of Mathematics, Statistics and Computer Science, Hyderabad; Public Health Foundation of India, Delhi, India; and current address for N. Scaglia: Instituto de Investigaciones Bioquímicas de La Plata, Facultad de Ciencias Médicas, UNLP-CONICET, Buenos Aires, Argentina.

Corresponding Author: Massimo Loda, Dana-Farber Cancer Institute, 450 Brookline Avenue, Dana 1536, Boston, MA 02115. Phone: 617-6324001; Fax: 617-632-4005; E-mail: massimo_loda@dfci.harvard.edu

doi: 10.1158/0008-5472.CAN-14-1490

(C)2014 American Association for Cancer Research. metabolome is considerably smaller than the number of genes, transcripts, or proteins, metabolomics may more clearly characterize altered cellular networks (4). Clinically, metabolic imaging technologies such as PET, can be used to monitor disease progression and drug response (5).

Genomic loss of the PTEN locus, leading to constitutive activation of the PI3K/AKT pathway, and $8 \mathrm{q}$ amplification, including the $M Y C$ gene, occurs in $30 \%$ to $70 \%$ and approximately $30 \%$ of prostate tumors, respectively (6), representing the most frequent genetic alterations in prostate cancer. Both activated AKT and in particular MYC overexpression faithfully reproduce the stages of human prostate carcinogenesis in genetically engineered mice $(7,8)$.

While MYC promotes glutaminolysis $(9,10)$, AKT activation is associated with enhanced aerobic glycolysis (the "Warburg effect"; ref. 11) and/or increased expression of glycolytic enzymes in different cell types, including prostate (12). However, the impact of these oncogenes (or the genomic alterations causing their activation) on the metabolome of human prostate tumors has not yet been elucidated.

\section{Materials and Methods}

Generation of AKT1- and MYC-overexpressing RWPE-1

Immortalized human prostate epithelial RWPE-1 cells were obtained from Novartis and confirmed to be nontumorigenic (growth in soft agar) before performing the experiments. RWPE-1 was authenticated by DDC Medical. Cells were 
infected with pBABE vector alone (RWPE-EV), myristoylated AKT1 (RWPE-AKT1) or MYC (RWPE-MYC). Briefly, cells were transduced through infection in the presence of polybrene $(8 \mu \mathrm{g} / \mathrm{mL})$, and retroviral supernatants were replaced with fresh media after 4 hours of incubation. Twenty-four hours later, puromycin selection $(1 \mu \mathrm{g} / \mathrm{mL})$ was started. Cells were grown in phenol red-free Eagle's Minimum Essential Medium (MEM) supplemented with $10 \% \mathrm{FBS}, 0.1 \mathrm{mmol} / \mathrm{L}$ nonessential amino acids, $1 \mathrm{mmol} / \mathrm{L}$ sodium pyruvate, and penicillinstreptomycin.

\section{Transgenic mice}

Ventral prostate lobes were isolated from 12- to 13-week-old MPAKT (FVB-Tg[Pbsn-AKT1]9Wrs/Nci; ref. 7) and Lo-Myc (FVB-Tg[Pbsn-MYC]6Key/Nci; ref. 8) transgenic mice and from age-matched wild-type mice $(\mathrm{FVB} / \mathrm{N})$ within 10 minutes after $\mathrm{CO}_{2}$ euthanasia. Animals' care was in accordance with institutional guidelines (Institutional Animal Care and Use Committee). MPAKT mice were generated and raised at the DanaFarber Cancer Institute's Facility (7). Lo-MYC and wild-type mice were obtained from the NCI Frederick mouse repository and raised at the Johns Hopkins University's Facility (13).

\section{Human prostate tissues}

Fresh-frozen, radical prostatectomy samples approved by the Institutional Review Board were obtained from the institutional tissue repository at the Dana-Farber Cancer Institute and Brigham and Women's Hospital (Boston, MA; 61 tumors and 25 normals).

The percentage of tumor was assessed by top and bottom frozen sections. To obtain $\geq 80 \%$ tumor purity, normal tissue was trimmed and the tumor sample was reembedded in optimal cutting temperature without thawing. Sections (two or three $8-\mu \mathrm{m}$ sections) were cut from these tissue blocks and DNA, RNA, and proteins were purified (AllPrep DNA/RNA Micro Kit; Qiagen Inc.). The remainder was processed for metabolite extraction (Supplementary Fig. S1C).

\section{Metabolite profiling}

RWPE-EV, RWPE-AKT1, and RWPE-MYC cells in monolayer culture were trypsinized for 4 minutes at $37^{\circ} \mathrm{C}$. Following trypsin neutralization with $10 \%$ FBS-supplemented MEM, cells were centrifuged, and cell pellets were washed twice with cold PBS before freezing. A recovery standard was added before the first step in the extraction process for quality control purposes. Aqueous methanol extraction was used to remove the protein fraction. The resulting extract was divided into fractions for analysis by UPLC/MS-MS (positive mode), UPLC/MS-MS (negative mode), and GC/MS. Samples were placed on a TurboVap (Zymark) to remove the organic solvent. Each sample was frozen and dried under vacuum (see also Supplementary Materials and Methods).

\section{Absolute quantitation of metabolites}

Fifty-six human prostate tissue samples (40 tumors and 16 normals) were used to validate the untargeted metabolite profiling. Oleic, arachidonic, and docosahexaenoic acids, creatine and 2-aminoadipic acid were measured using specific internal standards (see also Supplementary Materials and Methods). Absolute values were expressed as $\mu \mathrm{g} / \mathrm{g}$ tissue. Results were analyzed using the Mann-Whitney test, and significance was defined with $P<0.05$.

\section{mRNA expression analysis}

Total RNA was isolated from RWPE-EV, RWPE-AKT1, and RWPE-MYC cells (RNeasy Micro Kit; Qiagen Inc.), prostate tumors, and normal controls (AllPrep DNA/RNA Micro Kit; Qiagen Inc.). Real-time PCR was performed using custom micro fluidic cards (TaqMan Custom Arrays; Applied Biosystems). The list of the probes and primers is provided in Supplementary Text S1. One-sample $t$ test was applied using GraphPad Prism 5.0, and significance was defined with $P<0.05$ (see also Supplementary Materials and Methods).

\section{Immunohistochemistry}

Immunohistochemistry for MYC, stathmin, GLUT-1, and phosphoAKT1 was performed on formalin-fixed paraffinembedded tissue samples. Immunostaining of human tumors was scored microscopically counting the percentage of positive cells (higher or lower than 50\%). The entire section was evaluated and five representative areas at $\times 20$ magnification were counted for an average of 500 cells per section. The Fisher test was applied using GraphPad Prism 5.0, and significance was defined with $P<0.05$ (see also Supplementary Materials and Methods).

\section{Results and Discussion}

To focus on an oncogene-specific context while profiling the metabolic heterogeneity of prostate cancer, we integrated phosphorylated AKT1- or MYC-associated metabolomic signatures from prostate epithelial cells in monolayer culture, transgenic mouse prostate, and primary nonmetastatic prostate tumors (Fig. 1). We aimed at identifying patterns of metabolomic changes that were different for the two oncogenes but common for the datasets. Human tumors were assayed for phosphorylated AKT1 and MYC expression by immunoblotting. All of these tumors were pathologic stage T2, 22 high Gleason $(4+3$ or $4+4)$ and 38 low Gleason $(3+3$ or $3+4)$. Levels of phosphoAKT1 and MYC were not associated with the Gleason grade of the tumors (Supplementary Fig. S1A). K-means clustering of phosphorylated AKT1 and MYC densitometric values (Fig. 1C) was conducted to segregate four prostate tumor subgroups, that is, phosphoAKT1-high/MYChigh $(6 / 60,10 \%)$, phosphoAKT1-high/MYC-low (21/60, 35\%), phosphoAKT1-low/MYC-high (9/60, 15\%), and phosphoAKT1low/MYC-low (24/60, 40\%; Fig. 1C and Supplementary Fig. S1B).

To define differential metabolic reprogramming induced by the sole activation of AKT1 or overexpression of MYC, we performed mass spectrometry-based metabolomics of prostate epithelial nontransformed RWPE-1 cells genetically engineered with constructs encoding myristoylated AKT1 or MYC, and transgenic mice expressing human myristoylated AKT1 or MYC in the prostate (Fig. 1A and B). More than 50 metabolite sets (KEGG annotation-Supplementary Dataset S1) were 


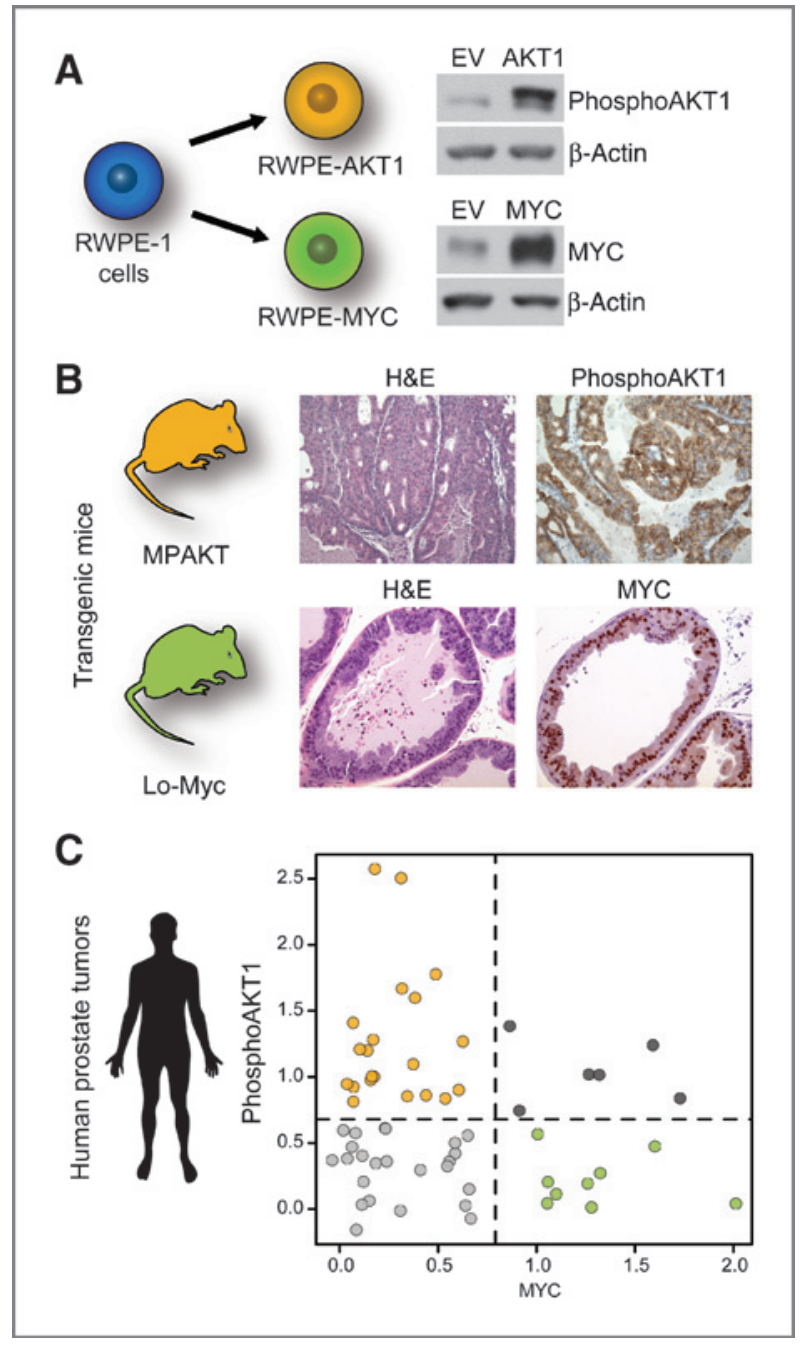

Figure 1. Integrative models of AKT or MYC-driven prostate tumorigenesis. Metabolomic profiling was performed on nontransformed prostate RWPE-1 cells (A) and mice (B) genetically engineered to overexpress myristoylated AKT1 or MYC, primary nonmetastatic prostate tumors (C), and controls. AKT1 and MYC overexpression is represented by orange and green, respectively. Blue, controls. A, phosphoAKT1 and MYC levels in RWPE-AKT1, RWPE-MYC, and control (RWPE-EV) cells are shown by immunoblots. B, both MPAKT and Lo-MYC transgenic mice exhibited prostate intraepithelial neoplasia at 11 to 13 weeks of age, as shown by hematoxylin and eosin (H\&E) staining. Overexpression of phosphoAKT1 and MYC was confirmed by immunohistochemistry. C, K-means clustering was used to segregate four prostate tumor subgroups, that is, phosphoAKT1-high/MYC-high (dark gray dots), phosphoAKT1-high/MYC-low (orange dots), phosphoAKT1-low/MYC-high (green dots), and phosphoAKT1-low/ MYC-low (light gray dots).

tested using single-sample Gene Set Enrichment Analysis (GSEA). A clear clustering of phosphoAKT1-high versus MYC-high samples was noticeable within the genetically engineered cell and mouse datasets, with phosphoAKT1-high being associated with the strongest phenotype in a distinct cluster compared with MYC-high and control samples that appeared closer together, yet recognizable as two subclusters (Fig. 2A and B). Human tumors fell in three clusters (defined by
Silhouette analysis), in which the phosphoAKT1-low/MYChigh tumors and the phosphoAKT1-high/MYC-low tumors differentially segregated (Fisher test, $P<0.01$; Fig. $2 \mathrm{C}$ ). Interestingly, although both RWPE-AKT1 and RWPE-MYC cells showed significant changes in intermediates of glucose metabolism and higher enrichment of the glycolysis set compared with RWPE-EV cells (Fig. 2A), only RWPE-AKT1 cells exhibited accumulation of lactate (aerobic glycolytic phenotype; Supplementary Fig. S2A). These results were even more pronounced in vivo, with exclusively the MPAKT mouse prostate samples being characterized by both high levels of lactate and enrichment of the glycolysis set (Fig. 2B and Supplementary Fig. S2A). When applied to primary nonmetastatic prostate tumors stratified by the expression levels of phospho-AKT1 and MYC, the pathway enrichment analysis revealed that MYChigh tumors have a negative enrichment of glycolysis compared with nontumoral prostate tissues (Fig. 2C and Supplementary Fig. S2A). Interestingly, normal prostate tissues may also be metabolically heterogeneous and exhibit a glycolytic phenotype (14), potentially attenuating the metabolic differences between normal and tumor tissue in phosphoAKT1-high tumors.

Next, we compared directly the AKT1 and MYC metabolic signatures (Supplementary Datasets S2 and S3). Pathway enrichment analysis by GSEA was used to determine which metabolic pathways were commonly enriched across the genetically engineered models and the prostate tumor subgroups defined above, specifically comparing AKT1-high with MYC-high background. Gene set-size-normalized enrichment scores (NES) from GSEA were used to determine the extent and direction of enrichment for each pathway in the three datasets. Five pathways with highly positive NES and two pathways with highly negative NES across and common to the datasets were defined as outliers (Fig. 3A and Supplementary Fig. S3A and S3B). These results link AKT1 activation with glycolysis and other glucose-related pathways, including the pentose phosphate shunt and fructose metabolism, and MYC overexpression with deregulated lipid metabolism (Fig. 3A and Supplementary Fig. S3C). On the one hand, enrichment of the glycerophospholipid, glycerolipid, and pantothenate/CoA biosynthesis metabolite sets, as well as higher levels of lipogenesisfeeding metabolites such as citrate, were distinctively associated with MYC overexpression in RWPE cells, suggesting a MYC-dependent deregulation of synthesis and/or turnover of membrane lipids. Interestingly, higher levels of both omega-3 (docosapentaenoate and docosahexaenoate) and omega-6 (arachidonate, docosadienoate, and dihomo-linolenate) fatty acids were found in Lo-MYC mice and in phosphoAKT1-low/ MYC-high prostate tumors relative to MPAKT mice and phosphoAKT1-high/MYC-low tumors (Supplementary Dataset S2). Prostate cells may use unsaturated, exogenous essential fatty acids early during transformation, perhaps as energy sources via oxidation (15).

As a validation of untargeted metabolomics, absolute concentrations of selected metabolites were measured. Oleic, arachidonic, and docosahexaenoic acids were validated in phosphoAKT1-high/MYC-low tumors $(n=14)$ and phosphoAKT1-low/MYC-high tumors $(n=5)$. Oleic acid can be 


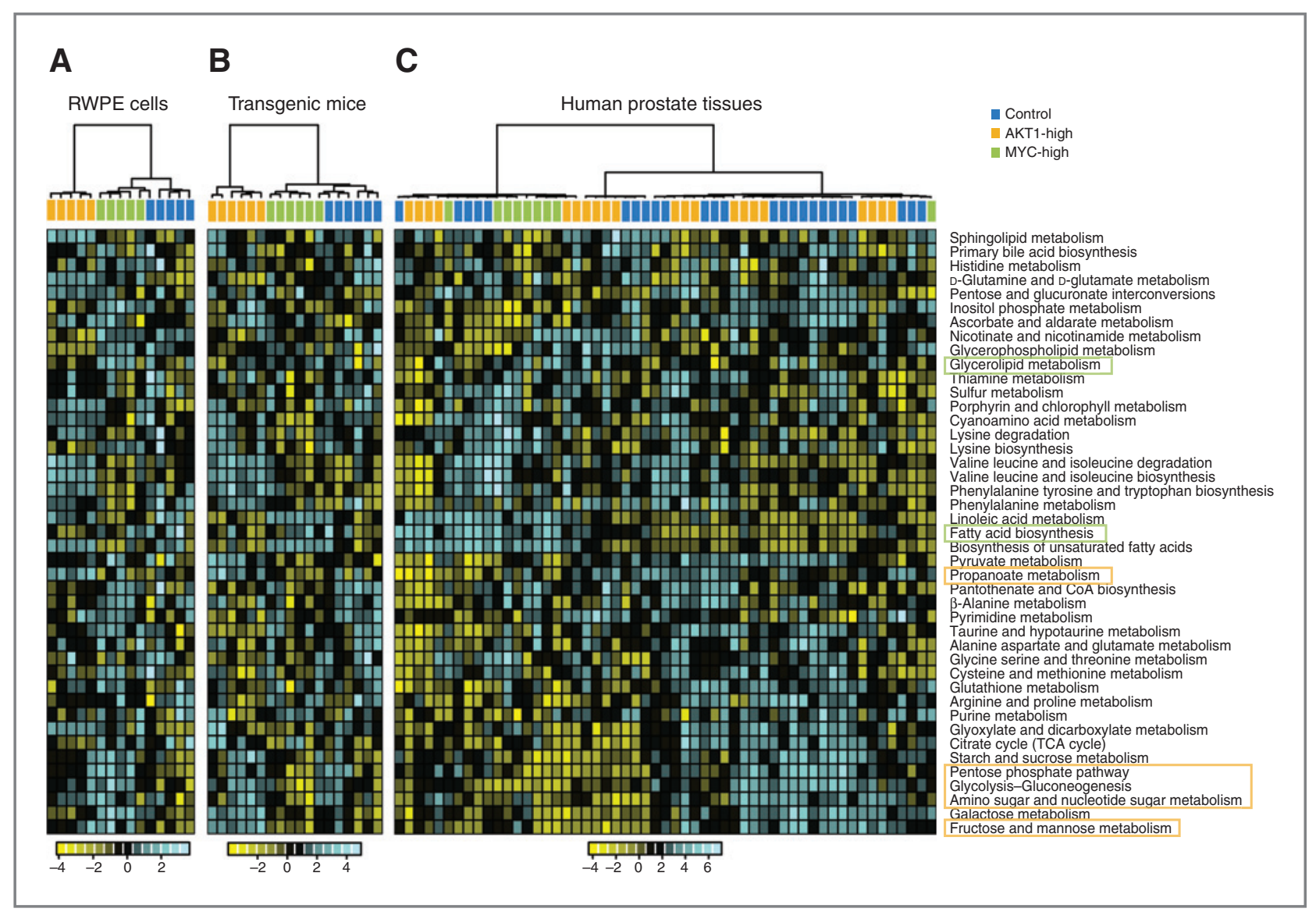

Figure 2. Metabolic pathway analysis in phosphoAKT1-high or MYC-high samples relative to controls. A-C, heatmap representation of NESs $(P<0.05)$ for KEGG pathways in individual samples of the three datasets (RWPE-1 cells, MPAKT and Lo-MYC mice, and human prostate tissues). Light blue/yellow colors are used to denote high/low enrichment, as in the respective color scales. Hierarchical clustering is used for unsupervised identification of the higher-level enrichment classes. The phenotypic labels of the samples (control, phosphoAKT1-high, and MYC-high) are indicated as a colored band on top of the heatmap, whereas the dendrogram represents the distances among them.

generated in the cell via desaturation of stearic acid by stearoylCoA delta-9-desaturase (SCD1). Consistent with the semiquantitative data, all of these fatty acids were present at a significantly higher concentration in MYC-high tumors (Fig. 3B). Additional validation of the untargeted metabolomics included the tumor-associated 2-aminoadipic acid, an intermediate of lysine metabolism, and creatine, which was increased in phosphoAKT1-high/MYC-low versus phosphoAKT1-low/ MYC-high tumors (Supplementary Fig. S4).

Next, we asked whether the metabolome changes associated with the oncogenic transformation of prostate epithelial cells are accompanied by transcriptional changes in key metabolic enzymes. Consistent with the metabolite profiling of RWPE-1 cells, glycolytic components such as the glucose transporter GLUT-1 and the hexokinase 2 were increased upon AKT1 overexpression/activation (Fig. 4A). As expected, downstream targets of AKT1 such as HIF $1 \alpha$ (hypoxia-inducible factor 1 ) and VEGF-A were induced in AKT1-overexpressing cells (Supplementary Fig. S5A). RWPE-MYC cells showed increased expression of two key enzymes of the glycerophospholipid metabolism, choline kinase alpha, and cholinephosphotransferase-1 (Fig. 4A). At the proteins level, hexokinase 2 was increased by
AKT1 activation, and choline kinase alpha was induced by MYC overexpression (Fig. 4B). Consistent with published data (10), MYC induced the expression of glutaminase, a glutaminolytic enzyme responsible for the conversion of glutamine into glutamate, at both the mRNA and the protein levels (Fig. $4 \mathrm{~A}$ and $\mathrm{B}$ ), resulting in an increased amount of glutamate relative to RWPE-EV. AKT1 activation strongly increased the expression of the neutral amino acid transporter ASCT2 (Fig. $4 \mathrm{~A}$ and $\mathrm{B}$ ). Interestingly, mRNA and protein expression of fatty acid synthase (FASN) was higher in RWPE-AKT1 and RWPEMYC cells compared with RWPE-EV cells (Supplementary Fig. S5A and S5C), as well as in prostate tumors compared with normal prostate tissue samples (Supplementary Fig. S5B and S5C). Although FASN expression can be induced downstream of AKT1 via mTORC1-mediated SREBP1 (sterol regulatory element-binding protein 1) activation, a link between increased de novo lipogenesis and aerobic glycolysis has been proposed in various tumor types $(16,17)$, suggesting a multifaceted role of FASN.

Sarcosine, an intermediate of the glycine and choline metabolism previously identified as a progression marker in prostate cancer (18), was increased exclusively in the prostate of 


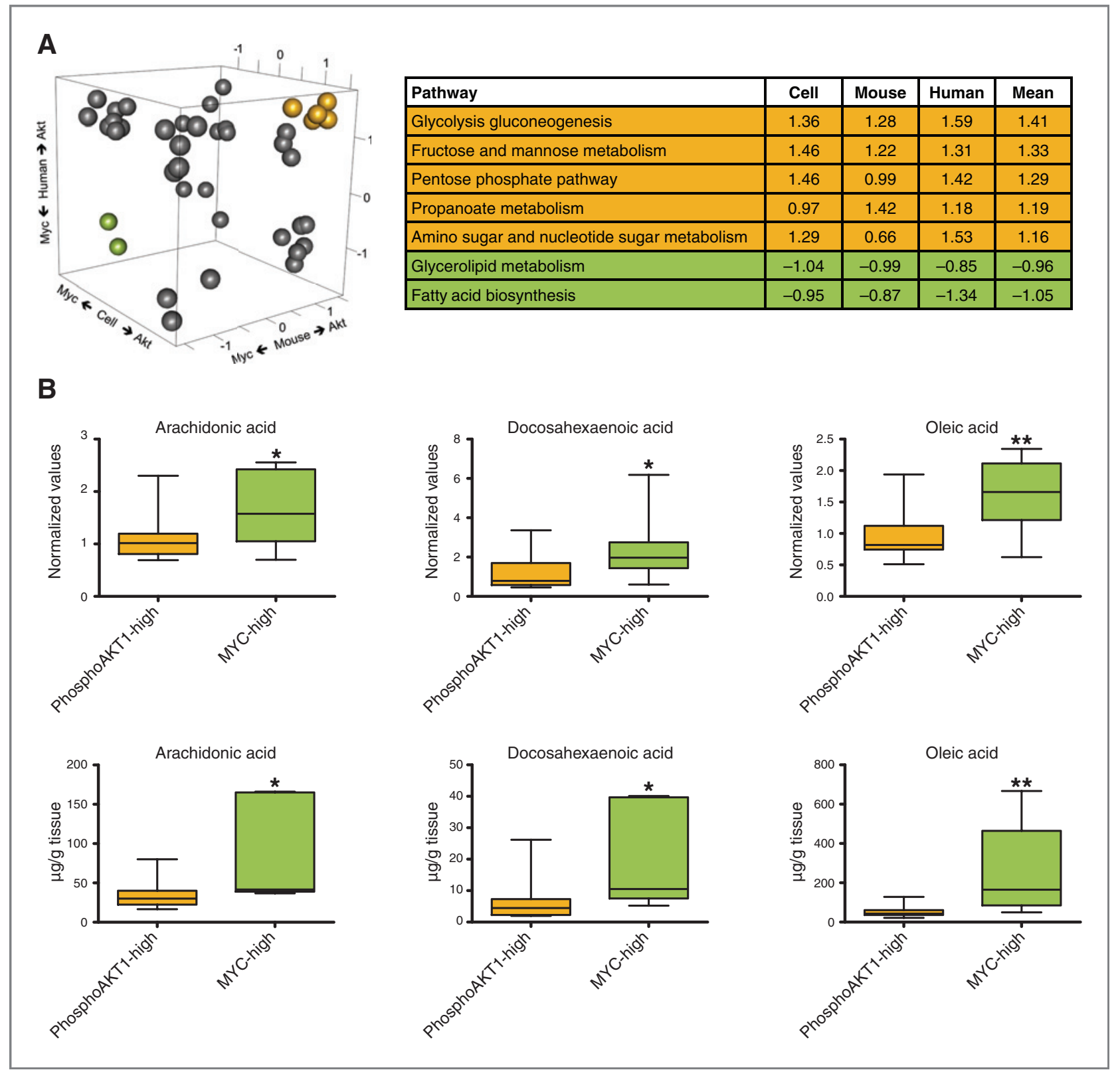

Figure 3. Overall differential metabolite set enrichments in phosphoAKT1-high versus MYC-high samples. A, simultaneous GSEA measurements in all three datasets (cultured cells, mouse prostate, and human tumors) are shown (left). This information is depicted as dots in three-dimensional space, where each dot represents a particular pathway and each dimension a dataset. Enrichment of a pathway in phosphoAKT1-high versus MYC-high samples or vice versa is defined by a positive or negative score, respectively. The top five positively enriched pathways (i.e., in phosphoAKT1-high samples) and the top two negatively enriched pathways (i.e., in MYC-high samples) in all three datasets, as identified with outlier analysis

(Supplementary Fig. S3), are shown as orange and green dots, respectively. NESs of the seven pathways identified as outliers in the three datasets and the average of these scores are shown per each set (KEGG pathway) on the right. B, semiquantitative (top) and absolute (bottom) measurements of arachidonic acid, docosahexaenoic acid, and oleic acid in phosphoAKT1-high/MYC-low (orange) and phosphoAKT1-low/MYC-high (green) tumor samples. The Mann-Whitney test was applied; ${ }^{*}, P<0.05$ and ${ }^{* *}, P<0.01$.

Lo-MYC mice (Supplementary Fig. S2B). Associated with the sarcosine increase were a concomitant elevation of the intermediate betaine and a decrease in glycine levels (Supplementary Fig. S2B). These results reflect a dysregulation of the sarcosine pathway by MYC.

To determine whether genomic alterations at the PTEN or MYC loci are predictive of active AKT1 or MYC overexpression in prostate tumors, we performed SNP arrays using genomic DNA isolated from the same sections of each tumor or nontumoral matched control sample assayed by immunoblotting (phosphorylated AKT1 and MYC). SNP arrays revealed that $20 \%$ of these tumors harbored $10 \mathrm{q}$ loss and $18 \%$ harbored $8 \mathrm{q}$ gain, including the MYC locus (Supplementary Fig. S6), whereas cooccurrence of PTEN loss and MYC copy gain was found in 


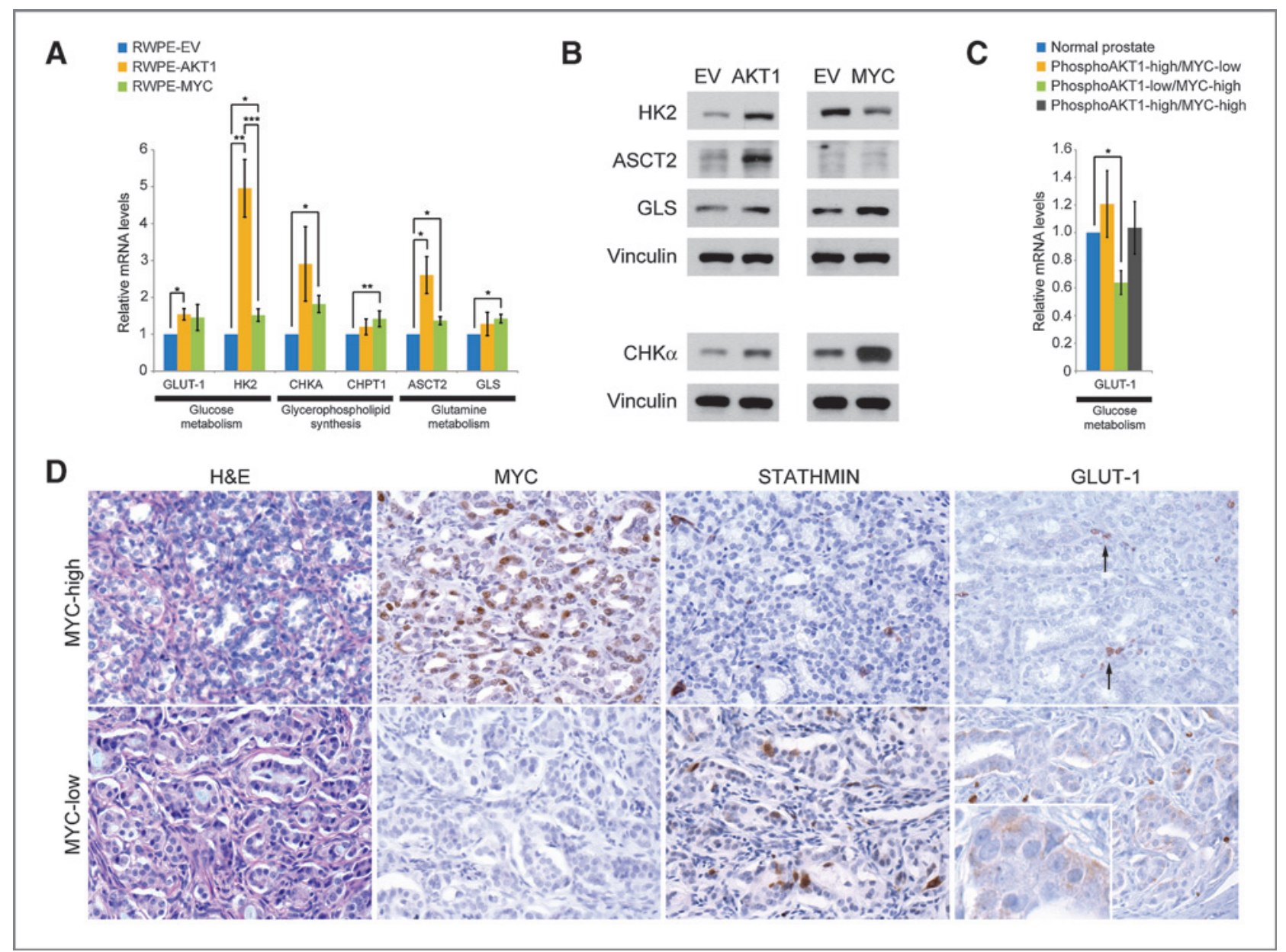

Figure 4. AKT1- and MYC-dependent regulation of metabolic gene expression. A, relative qPCR analysis of mRNA levels of genes of glucose, glycerophospholipid, and glutamine metabolism in RWPE-AKT1 (orange bars) and RWPE-MYC (green bars) cells normalized to RWPE-EV (blue bars) cells. B, immunoblotting of hexokinase 2 (HK2), the glutamine transporter ASCT2, glutaminase (GLS), and choline kinase alpha (CHK $\alpha$ ) in RWPE-AKT1, RWPE-MYC, and RWPE-EV cells. C, relative qPCR analysis of mRNA levels of the glucose transporter GLUT-1 in phosphoAKT1-high/MYC-low (orange bar; $n=13$ ), phosphoAKT1-low/MYC-high (green bar; $n=5$ ), and phosphoAKT1-high/MYC-high (dark gray bar; $n=3$ ) prostate tumors versus normal prostate samples (blue bar; $n=9$ ). D, hematoxylin and eosin (H\&E) and immunohistochemical staining for MYC, stathmin (an AKT downstream target used as a surrogate of AKT activity), and GLUT-1 in representative cases of phosphoAKT1-low/MYC-high and phosphoAKT1-high/MYC-low prostate tumors. Red cells (arrows) represent a positive control for GLUT-1 staining. One-sample $t$ test was performed using average fold change of at least three experiments (A) or samples (C); ${ }^{*}$ $P<0.05 ;{ }^{* *}, P<0.01 ;$ and ${ }^{* * *}, P<0.001$.

$3 \%$ of tumors, consistent with published data (19). Importantly, the genomic alterations accounted for $26 \%(7 / 27)$ of phosphoAKT1-high tumors and for $13 \%(2 / 15)$ of MYC-high tumors (Supplementary Fig. S6), as expected from previous reports (20).

Finally, to identify unique mRNA expression changes in phosphoAKT1-high/MYC-low and phosphoAKT1-low/MYChigh prostate tumors, we performed a qPCR-based expression profiling analysis of 28 metabolic genes (Supplementary Fig. S5D) in tumor relative to normal prostate tissues. Consistent with the MYC-dependent negative enrichment for the glycolytic pathway (Fig. 2C and Supplementary Fig. S2A), high MYC expression in a phosphoAKT1-low context in human tumors was associated with decreased mRNA expression of GLUT-1 (Fig. 4C). This finding was specific to the MYC-high tumors and not generalizable to all tumors versus normal prostate tissues
(Supplementary Fig. S5B). Also, no decrease in GLUT-1 expression was found in phosphoAKT1-high/MYC-high tumors (Fig. 4C). A significant association between GLUT-1 high expression and phosphoAKT1-high status was found by immunohistochemistry in a subset of this cohort (Fig. 4D and Supplementary Fig. S5C). Seven of 14 phosphoAKT1-low tumors were MYC-high, and only 14\% (1/7) of these showed high GLUT-1, whereas $85 \%$ (6/7) had low or no GLUT-1 expression (Fig. 4D). Altogether, these results suggest that AKT1 activation may be critical to maintain high GLUT-1 levels in prostate cancer cells, and that AKT1-independent MYC activation can potentially affect glucose uptake in prostate tumors.

In summary, our data demonstrate that individual prostate tumors have distinct metabolic phenotypes, resulting from their genetic complexity, and reveal a novel potential metabolic role for MYC in prostate cancer. The evidence provided 
links AKT1 or MYC activation with differential deregulation of glucose-related pathways as well as lipid metabolism in human prostate cancer. To our knowledge, this is the first report of oncogene-associated metabolic signatures as the result of an integrative analysis of cultured cells, mouse models, and human tumors. This opens novel avenues for the metabolic imaging and therapeutic targeting of patients with prostate cancer.

\section{Disclosure of Potential Conflicts of Interest}

No potential conflicts of interest were disclosed.

\section{Authors' Contributions}

Conception and design: C. Priolo, M. Loda

Development of methodology: C. Priolo, S. Pyne, M. Loda

Acquisition of data (provided animals, acquired and managed patients, provided facilities, etc.): C. Priolo, J. Rose, G. Zadra, D. Schultz, N. Scaglia, J. McDunn, A.M. De Marzo, M. Loda

Analysis and interpretation of data (e.g., statistical analysis, biostatistics, computational analysis): C. Priolo, S. Pyne, E.R. Regan, S. Cacciatore, N. Scaglia, J. McDunn, M. Loda

\section{References}

1. Bardella C, Pollard PJ, Tomlinson I. SDH mutations in cancer. Biochim Biophys Acta 2011;1807:1432-43.

2. Ward PS, Patel J, Wise DR, Abdel-Wahab O, Bennett BD, Coller HA, et al. The common feature of leukemia-associated IDH1 and IDH2 mutations is a neomorphic enzyme activity converting alpha-ketoglutarate to 2-hydroxyglutarate. Cancer Cell 2010;17:225-34.

3. Christofk HR, Vander Heiden MG, Harris MH, Ramanathan A, Gerszten RE, Wei R, et al. The M2 splice isoform of pyruvate kinase is important for cancer metabolism and tumour growth. Nature 2008:452:230-3.

4. Trock BJ. Application of metabolomics to prostate cancer. Urol Oncol 2011;29:572-81.

5. Schmid DT, John H, Zweifel R, Cservenyak T, Westera G, Goerres GW, et al. Fluorocholine PET/CT in patients with prostate cancer: initial experience. Radiology 2005;235:623-8.

6. Taylor BS, Schultz N, Hieronymus H, Gopalan A, Xiao Y, Carver BS, et al. Integrative genomic profiling of human prostate cancer. Cancer Cell 2010;18:11-22.

7. Majumder PK, Yeh JJ, George DJ, Febbo PG, Kum J, Xue Q, et al. Prostate intraepithelial neoplasia induced by prostate restricted Akt activation: the MPAKT model. Proc Natl Acad Sci U S A 2003;100: 7841-6.

8. Ellwood-Yen K, Graeber TG, Wongvipat J, Iruela-Arispe ML, Zhang J, Matusik R, et al. Myc-driven murine prostate cancer shares molecular features with human prostate tumors. Cancer Cell 2003;4:223-38.

9. Wise DR, DeBerardinis RJ, Mancuso A, Sayed N, Zhang XY, Pfeiffer $\mathrm{HK}$, et al. Myc regulates a transcriptional program that stimulates mitochondrial glutaminolysis and leads to glutamine addiction. Proc Natl Acad Sci U S A 2008;105:18782-7.

10. Gao P, Tchernyshyov I, Chang TC, Lee YS, Kita K, Ochi T, et al. c-Myc suppression of $\mathrm{miR}-23 \mathrm{a} / \mathrm{b}$ enhances mitochondrial glutaminase expression and glutamine metabolism. Nature 2009;458:762-5.

11. Warburg O. On the origin of cancer cells. Science 1956;123:309-14.
Writing, review, and/or revision of the manuscript: C. Priolo, S. Pyne, J. McDunn, A.M. De Marzo, M. Loda

Administrative, technical, or material support (i.e., reporting or organizing data, constructing databases): C. Priolo, C. Photopoulos, M. Loda

Study supervision: C. Priolo, M. Loda

\section{Acknowledgments}

The authors thank Matthew Vander Heiden for critical review of the article and to William C. Hahn for providing plasmids.

\section{Grant Support}

This work was supported by RO1CA131945, P50 CA90381, the Prostate Cancer Foundation, and philanthropic funds from Nuclea Biomarkers (Pittsfield, MA; M. Loda); the P.A.R.T. Investigatorship in Prostate Cancer Award from the Lank Center/Dana-Farber Cancer Institute and a Friends of Dana-Farber fund (C. Priolo); the Ramalingswami Fellowship from DBT, MoS\&PI and DST (CMS Project SR/SA/MS:516/07; 21/04/2008), India (S. Pyne); the Patrick C. Walsh Prostate Cancer Research Fund Award (A.M. De Marzo); and the Fondazione Italiana per la Ricerca sul Cancro, Italy (S. Cacciatore).

Received May 20, 2014; revised September 15, 2014; accepted October 2, 2014; published OnlineFirst October 16, 2014.

12. Majumder PK, Febbo PG, Bikoff R, Berger R, Xue Q, McMahon LM, et al. mTOR inhibition reverses Akt-dependent prostate intraepithelial neoplasia through regulation of apoptotic and HIF-1-dependent pathways. Nat Med 2004;10:594-601.

13. Iwata T, Schultz D, Hicks J, Hubbard GK, Mutton LN, Lotan TL, et al MYC overexpression induces prostatic intraepithelial neoplasia and loss of Nkx3.1 in mouse luminal epithelial cells. PLoS ONE 2010;5: e9427.

14. Xu Q, Majumder PK, Ross $K$, Shim $Y$, Golub TR, Loda $M$, et al Identification of prostate cancer modifier pathways using parental strain expression mapping. Proc Natl Acad Sci U S A 2007;104: 17771-6.

15. Carracedo A, Cantley LC, Pandolfi PP. Cancer metabolism: fatty acid oxidation in the limelight. Nat Rev Cancer 2013;13:227-32.

16. Menendez JA. Fine-tuning the lipogenic/lipolytic balance to optimize the metabolic requirements of cancer cell growth: molecular mechanisms and therapeutic perspectives. Biochim Biophys Acta 2010;1801:381-91.

17. Bhatt AP, Jacobs SR, Freemerman AJ, Makowski L, Rathmell JC, Dittmer DP, et al. Dysregulation of fatty acid synthesis and glycolysis in non-Hodgkin lymphoma. Proc Natl Acad Sci U S A 2012;109: 11818-23.

18. Sreekumar A, Poisson LM, Rajendiran TM, Khan AP, Cao Q, Yu J, et al Metabolomic profiles delineate potential role for sarcosine in prostate cancer progression. Nature 2009;457:910-4.

19. Clegg NJ, Couto SS, Wongvipat J, Hieronymus H, Carver BS Taylor BS, et al. MYC cooperates with AKT in prostate tumorigenesis and alters sensitivity to mTOR inhibitors. PLoS ONE 2011;6:e17449.

20. Koh CM, Bieberich CJ, Dang CV, Nelson WG, Yegnasubramanian S, De Marzo AM. MYC and prostate cancer. Genes Cancer 2010;1: 617-28. 


\section{Cancer Research}

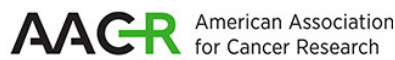

The Journal of Cancer Research (1916-1930) | The American Journal of Cancer (1931-1940)

\section{AKT1 and MYC Induce Distinctive Metabolic Fingerprints in Human Prostate Cancer}

Carmen Priolo, Saumyadipta Pyne, Joshua Rose, et al.

Cancer Res 2014;74:7198-7204. Published OnlineFirst October 16, 2014.

Updated version Access the most recent version of this article at: doi:10.1158/0008-5472.CAN-14-1490

Supplementary Access the most recent supplemental material at:

Material http://cancerres.aacrjournals.org/content/suppl/2014/10/16/0008-5472.CAN-14-1490.DC1

Cited articles This article cites 20 articles, 5 of which you can access for free at: http://cancerres.aacrjournals.org/content/74/24/7198.full\#ref-list-1

Citing articles This article has been cited by 13 HighWire-hosted articles. Access the articles at: http://cancerres.aacrjournals.org/content/74/24/7198.full\#related-urls

E-mail alerts Sign up to receive free email-alerts related to this article or journal.

Reprints and To order reprints of this article or to subscribe to the journal, contact the AACR Publications Department at Subscriptions pubs@aacr.org.

Permissions To request permission to re-use all or part of this article, use this link http://cancerres.aacrjournals.org/content/74/24/7198.

Click on "Request Permissions" which will take you to the Copyright Clearance Center's (CCC) Rightslink site. 